\title{
Condições de radioproteção dos consultórios odontológicos
}

\author{
Radioprotection in dentistry offices
}

M aria de Fátima Batista de M elo 1,2

Saulo Leonardo Sousa M elo ${ }^{1}$

${ }^{1}$ Departamento de

Odontologia, Centro de

Ciências Biológicas e da

Saúde, UniversidadeFederal

deSergipe. RuaCláudio

Batista $s / n$, Sanatório.

49060-100 Aracaju SE.

slsmelo@uol.com.br.

${ }^{2}$ Curso de O dontologia,

UniversidadeTiradentes.
Abstract In 1998, theBrazilian M inistry of $\mathrm{H}$ ealth established the Guidelines for Protection in M edical and $O$ dontological Radiography, aimed at ensuring the quality of radiological procedures provided to the population as well as radiological protection for the health professionals. Thepurpose of this study was to verify the current situation in the dental offices with regard to the technical specifications and the adopted protective measures, using a questionnaire including questions about equipments, techniques and radiological protection. The investigated universe consisted of the surgeon dentists of the city of Aracaju using radiographic examination as a routine, resulting in a final sample of 103 professionals. Theresults showed that a high percentage of these professionals ignore the current norms $(64,1 \%)$ as well as the technical specifications of their equipment ( $51,5 \%$ ignored the peak kilovoltage and $57,3 \%$ the milliamperage of their equipment), besides examining theradiographs visually (67\%) As positive behaviors, the paralleling technique was preferred by $62,1 \%$ and the lead apron was used by $98,1 \%$. We concludethat, despitethelimited knowledgeabout the Guidelines, some measures are being correctly adopted but that the dentists haveto bemoreaware of the necessary protective measures. Key words Radiology, Standards, Radiation protection, Radiography, Dental
Resumo Em 1998, o M inistério da Saúde estabe leceu as Diretrizes de Proteção Radiológica em Radiodiagnóstico M édico e 0 dontológico, visando garantir a qualidade nos procedi mentos prestados à população e assegurar a proteção radiológica aos profissionais da saúde. 0 objetivo desta pesquisa foi verificar a situação dos consultórios odontológicos quanto às especificações técnicas preconizadaseàsmedidas de proteção adotadas, utilizandose um questionário com itens sobre equi pamentos, técnicas eproteção radiológica. 0 universo foi composto pelos cirurgiões-dentistas do município de Aracaju, Sergipe, que executam o exameradiográfico como rotina, sendo a amostra final de 103 profissionais. Dentre os resultados encontrados, destacam-se 0 alto índice de profissionais que desconhecem as normas atuais $(64,1 \%)$ e as especificações técnicas de seus equipamentos (51,5\% desconhecem a quilovoltagem e $57,3 \%$, a miliamperagem), como também aqueles que utilizam o mé todo visual de revelação (67\%). Como condutas positivas, $62,1 \%$ dos pesquisados fazem uso da técnica do paralelismo e $98,1 \%$ utilizam a proteção plumbífera no paciente. Com isso, conclui-se que apesar de uma minoria conhecer as Diretrizes de Proteção, algumas medidas estão sendo corretamente adotadas; todavia, uma melhor conscientização se faz necessária.

Palavras-chave Radiologia, Normas, Proteção radiológica, Radiografia dentária 


\section{Introdução}

O exame radiográfico é um valioso método auxiliar de diagnóstico nos diversos procedimentos adotados pelo cirurgião-dentista, sendo utilizado em qualquer manobra clínica pré, trans ou pós-operatória.

Desde a descoberta dos raios $X$ por Wilhelm Conrad Röntgen, em novembro de 1895, várias pesquisas vêm enaltecendo a importância desta radiação para a humanidade, bem como suas ações deletérias devido à ação ionizante. Em nosso organismo, a ação ionizantesefaz sentir especialmente nos cromossomos, cujos efeitos se manifestam durantea divisão celular, causando a evolução anormal ou morte da célula ${ }^{1}$, sendo os principais riscos associados com baixas doses de radiação: 0 câncer, as mutações e as anomalias congênitas².

As autoridades afirmam que qualquer dose de radiação tem potencial de produzir danos, mas, de acordo com a literatura, os efeitos potenciais da radiação advinda de aparelhos de raios $X$ odontológico são mínimos, sendo o risco de indução de um câncer fatal por uma radiografia panorâmica de 1:1.000.000 e por uma radiografia intra-oral de 1:10.000.0003. Todavia, apesar de o risco ser muito baixo, é importante considerar que os efeitos da radiação podem ser acumulativos; por isso, todos os dentistas têm a responsabilidade profissional com seus pacientes, sua equipeeasi mesmo deminimizar todos os riscos que possam estar associados à radiação $0^{3,4}$.

A conscientização dessa ação deletéria dos raios $X$, através de efeitos somáticos ou genéticos, levou os pesquisadores a buscar medidas de segurança na aplicação desta radiação, surgindo na Alemanha em 1913 as primeiras regras para o uso dos raios $X^{5}$. Oito anos depois, em 1921, foram relatadas as primeiras recomendações de proteção radiológica pelo "British X-ray and Radium Committee". Todavia, foi somente em 1928 que foram estabelecidas as primeiras normas de proteção pela "International Commission of Radiological Protection"6.

Seguindo a tendência mundial, em 1 dejunho 1998, o M inistério da Saúde do Brasil estabeleceu a portaria $n$ - 453 intitulada "Diretrizes de Proteção Radiológica em Radiodiagnóstico M édico e Odontológico"7 , que se preocupa com a necessidade de garantir a qualidade nos procedimentos de radiodiagnóstico prestados à população, assim como assegurar os requisitos mínimos de proteção radiológica aos profissionais da área de saúde.

A preocupação com as medidas de radiopro- teção adotadas pelos cirurgiões-dentistas tem levado diversos pesquisadores a estudar 0 assunto em todo o mundo, assim como no Brasil, buscando avaliar os cuidados adotados para minimizar a exposição aos raios $X$, como também 0 uso dessa radiação nos consultórios e serviços públicos em geral. A metodologia aplicada nesses estudos consiste na elaboração de questionários, contendo diversas perguntas fechadas e/ ou abertas sobre 0 assunto em questão.

Assim, constituiu o objetivo desta pesquisa verificar a situação atual dos consultórios odontológicos do município de Aracaju, quanto às especificações técnicas preconizadas pelo M inistério da Saúde, no que diz respeito às condições de instalação, manutenção e uso dos aparelhos de raios $\mathrm{X}$ e às medidas de proteção adotadas pelos cirurgiões-dentistas entrevistados.

\section{Método}

Este estudo foi aprovado pelo Comitê de Ética em Pesquisa em Seres Humanos da Universidade Federal de Sergipe (UFS), segundo a Resolução n 196/96 do Conselho Nacional de Saúde.

Tratou-se de uma pesquisa de campo, de caráter descritivo, com uma abordagem quantitativa através de levantamento de dados, utilizando a técnica de coleta de informações baseada num questionário com perguntas fechadas, elaborado especial mente para este fim eque foi entregue e recolhido pessoalmente. A partir desse questionário, buscou-se avaliar de forma simples, porém ampla, o conhecimento e o comportamento dos entrevistados em relação às normas de proteção radiológica vigentes.

0 universo desta pesquisa foi composto pelos cirurgiões-dentistas do município de Aracaju, Sergipe, que executam o exame radiográfico na sua rotina clínica, sendo a amostra, inicialmente, compreendida por $30 \%$ de todos os cirurgiões-dentistas inscritos entre 1974 e 2004 no Conselho Regional deO dontologia - Secção Sergipe (275 dos 917 profissionais), considerando um nível de confiança de $95 \%$ (erro relativo de $5 \%)$. Contudo, dos profissionais que constituíam a amostra planejada, foi conseguido um retorno de 103 questionários, sendo o nível deconfiança de $92 \%$ (erro relativo $8 \%$ ).

A amostra foi selecionada a partir do banco de dados fornecido pelo Conselho Regional de Odontologia, na qual, depois de feitas as devidas exclusões, foram sorteados de forma aleatória e através do número de inscrição nesse Conselho. 
Foram considerados critérios de exclusão do sujeito: ser especialista em radiologia; trabalhar em centros de diagnóstico por imagem; não atuar no município visado (Aracaju); falecimento; e não exercer a profissão.

A amostra final representa os profissionais que, após esclarecimento dos objetivos da pesquisa, dispuseram-se a participar do estudo, assinando o Termo de Consentimento Livre e Esclarecido erespondendo o questionário, durante as visitas do pesquisador.

Os dados colhidos nos questionários foram tabulados para serem analisados, sendo os re sultados organizados como um banco de dados no programa Microsoft Excel $2003^{\circledR}$ e expressos sob a forma de percentagem. As conclusões basearam-senuma combinação de análise descritiva e discussão, sob o ponto de vista clínico, dos vários itens abordados.

\section{Resultadosediscussão}

A preocupação com as medidas de radioproteção adotadas pelos cirurgiões-dentistas tem levado diversos pesquisadores a estudar 0 assunto em todo o mundo, como Farman \& Nortjé, na África do Sul; Farman \& Hines ${ }^{9}$, na América do Norte; Smith ${ }^{5}$, naAlemanha; M onsour et al. ${ }^{10}$, na Austrália; Mageean ${ }^{11}$, na Irlanda do Norte; Goren et al. ${ }^{12}$ e Geist \& Katz $^{13}$, nos Estados Unidos da América; Svenson \& Petersson ${ }^{14}$, Svenson et al. ${ }^{15}$ e Svenson et al. ${ }^{16}$, na Suécia; Mutyabule \& Whaites ${ }^{17}$, em Uganda; Jacobs et al. ${ }^{18}$, na Bélgica; Stavrianou et al. ${ }^{19}$, na Grécia; e llgüy et al. ${ }^{20}$, na Turquia.

No Brasil, alguns trabalhos de relevância são os de M elo et al ${ }^{21}$, em São Paulo, capital; Capelozza \& Álvares ${ }^{22}$, em Bauru, São Paulo; Garcez FiIho et al. ${ }^{23}$, em Aracaju, Sergipe; Salineiro \& Capelozza ${ }^{24}$, em Araçatuba, São Paulo; Bóscolo et al. ${ }^{25}$, em M inas Gerais; Spyrides et al. ${ }^{26}$, no Rio de Janeiro, capital; Barbosa \& Gewehr ${ }^{27}$, nos estados do Sul; e Silveira et al ${ }^{28}$, em O linda, Pernambuco.

Contudo, após mais de oito anos da publicação das Diretrizes de Proteção Radiológica no Diário Oficial da União ${ }^{7}$, percebe-se que muitos erros são cometidos na prática diária nos consultórios odontológicos, como também a existência de muitos questionamentos por parte dos profissionais.

$\mathrm{N}$ a presente pesquisa, que emprega somente observação indireta através do uso de questionário auto-aplicável, não foi necessário a realização de estudo piloto, visto que a metodologia adotada, semelhante à da mai oria dos pesquisadores revisados, está internacionalmente validada. A penas na elaboração do questionário houve pequena adaptação dos itens abordados outrora, de forma a adequá-los à realidade local.

Em relação ao conhecimento pelos profissionais das normas vigentes e à inspeção da Vigilância Sanitária quanto ao uso da radiação no consultório odontológico, encontrou-seque $65 \%$ dos profissionais haviam recebido a visita da Vigilância, sendo que apenas 35,9\% afirmaram ter conhecimento das normas vigentes. Dados semelhantes foram encontrados por Barbosa \& Gewehr ${ }^{27}$, no qual apenas $40 \%$ conheciam o conteúdo da portaria $n^{\circ} 453^{7}$. Entretanto, esses valores diferem dos encontrados por M elo et al. ${ }^{21}$, no qual $54 \%$ conheciam as recomendações internacionais do período, edos estudos de Garcez Filho et al..$^{23} \mathrm{em}$ que, de acordo com os autores, $88,6 \%$ dos entrevistados conheciam, ainda que superficialmente, as normas vigentes na época.

De acordo com a portaria $n^{\circ} 453^{7}$, art. $5 \S 10$, alínea a, em radiografias intrabucais, a tensão no tubo de raios $X$ deve ser maior ou igual a $50 \mathrm{kVp}$, preferencialmente maior que $60 \mathrm{kV}$. Dos profissionais que souberam informar a respeito, foi encontrado que $34 \%$ dos aparelhos utilizados possuíam tensão maior que $60 \mathrm{kVp}, 11,6 \%$ em $60 \mathrm{kVp}$ e 2,9\% em $50 \mathrm{kVp}$. Os valores da corrente destes aparel hos variaram entre 6 e $10 \mathrm{~mA}$, segundo informação dos 42,7\% que souberam informar.

Pelo fato de a tensão e a corrente do equipamento influenciar diretamente no poder de penetração da radiação e no contraste da imagem gerada11,29, é de relevante preocupação o fato de muitos cirurgiões-dentistas não conhecerem a tensão $(51,5 \%)$ e e corrente $(57,3 \%)$ de seus equipamentos, sendo esse fato também observado por Spyrides et al. ${ }^{26}$, em $62 \%$ dos entrevistados; por Mutyabule\& Whaites ${ }^{17}$, em $65 \%$ deles; epor Ilgüy et al. ${ }^{20}$, em $86,9 \%$.

Outro fator que influencia no tempo de exposição e na qualidade da imagem é a sensibilidade do filme radiográfico. Segundo as Diretrizes $^{7}$, deve ser usado o receptor de imagem mais sensível que possa fornecer o nível de contrastee detalhe necessários. A pesar de ainda ser encontrado filmes de sensibilidade D, hoje no mercado estão disponíveis filmes de sensibilidade $E$, que reduzem o tempo de exposição em $52 \%$, comparando-se com o de sensibilidade D; como também são comercializadas películas com sensibilidade $F$, que necessitam de $20 \%$ menos tempo de exposição que as de sensibilidade $\mathrm{E}^{19}$. N a presente pesquisa, foi encontrado que $50,5 \%$ dos pro- 
fissionais utilizam filmes de sensibilidade $\mathrm{E}$ e $20,4 \%$, de sensibilidade $E / F$. Apenas quatro cirurgiões-dentistas $(3,9 \%)$ continuam utilizando películas de bai xa velocidade (sensibilidadeD).

Valores semelhantes foram encontrados por Farman \& Hines ${ }^{9} ;$ M onsour et al. ${ }^{10} ;$ Capelozza \& Álvares ${ }^{22}$; Svenson $\&$ Peterssonn ${ }^{14}$; Svenson et al. ${ }^{15}$; Spyrides et al. ${ }^{26} ;$ Geist $\&$ Katz ${ }^{13}$; Tosoni et al..$^{30}$; e Jacobs et al. ${ }^{18}$. A penas os resultados de Goren et al. ${ }^{12}$ divergiram, nos quais somente $13 \%$ dos profissionais de N ova Iorque (Estados Unidos) utilizavam filmes de sensibilidade E. Novamente, é destacável o número de entrevistados que não souberam informar o tipo de filmeusado (25,2\%), em concordância com os achados de M utyabule $\&$ Whaites ${ }^{17}$, que encontraram $40 \%$, sendo menor que os $65,8 \%$ relatados por Ilgüy et al. ${ }^{20}$.

Pelos melhores resultados obtidos e menores irradiações teciduais que oferece, em radiografias intrabucais, deve-se utilizar, preferencialmente, a técnica do paralelismo com localizadores longos, dispositivos de alinhamento (posicionadores) e prendedores de filme, de modo a evitar que o paciente tenha que o segurar (art. $5 \S 8$, alínea d) ${ }^{7}$. Observou-se que $62,1 \%$ dos entrevistados utilizam essa técnica adaptada no seu diaa-dia, demonstrando uma adequação às exigências atuais, quando comparado ao valor de $81,4 \%$ encontrado por Garcez Filho et al. ${ }^{23} \mathrm{em} 1990$.

Valor semelhante foi encontrado por Goren et al. ${ }^{12}$; entretanto, a técnica da bissetriz foi mais utilizada nos achados de M elo et al..$^{21}$, em mais de $80 \%$; M onsour et al. ${ }^{10}$, em 75\%; Spyrides et al..$^{26}$, em $71 \%$; Mutyabule \& Whaites ${ }^{17}$, em $80 \%$; e Tosoni et al. ${ }^{30}$, em $73,9 \%$. 0 fato dea maioria $(62,1 \%)$ utilizar posicionador, garantindo uma melhor perpendicularidade do feixeprimário em relação ao filme, podeter influenciado paraqueo número de repetições dos exames radiográficos variasse numa média de até duas repetições por dia de trabalho.

0 art. $5 \S 9$, alíneas c e d, das Diretrizes ${ }^{7}$ faz referência ao posicionamento adequado do operador ou qualquer membro da equipe, em relação à direção do feixe primário, e a não estabilização do cabeçote localizador e/ou do filme radiográfico durante as exposições. Portanto, a estabilidade do cabeçote deve ser verificada constantemente, evitando movimento depois de posicionado, o que poderesultar em perda de nitidez e meia lua, e no caso de ser necessário manter 0 filme na boca do paciente, isto deve ser feito por um acompanhante do mesmo ${ }^{29}$.

$\mathrm{Na}$ presente pesquisa, foi encontrado que $40,8 \%$ dos entrevistados pede ao paciente para segurar o cabeçote a fim de estabilizá-lo e que a maioria $(76,8 \%)$ utiliza o posicionador para manter o filmena posição correta, apesar de $9,7 \%$ manter o filme na boca do paciente quando este não conseguia fazêlo. Ao contrário dos dados encontrados por $\mathrm{M}$ elo et al. ${ }^{21}$, que relataram que considerável parte dos entrevistados mantinham pessoalmente o filme na boca do paciente ou alguém da equipe o fazia (22\%), M onsour et al. ${ }^{10}$ encontraram que $40 \%$ dos profissionais o faziam; Garcez Filho et al. ${ }^{23}$, 37,1\%; Salineiro \& Capelozza ${ }^{24}, 5 \%$; Jacobs et al. ${ }^{18}, 8 \%$; el lgüy et al. ${ }^{20}$ relataram $16,8 \%$.

Deacordo com as Diretrizes de Proteção ${ }^{7}$, em seu art. $5 \S 7$ alínea e, os equipamentos para radiografias intrabucais devem possuir um localizador de extremi dade de saída aberta para posicionar o feixe e limitar a distância foco-pele. Além disso, um método efetivo de redução da dose de radiação é manter a área irradiada a menor possível, compatível com o tamanho do objeto radiografado, sendo isso conseguido com o uso de cones localizadores longos 4 . Em Aracaju, a maioria dos cirurgiões-dentistas entrevistados (83,5\%) tem em seus consultórios equipamentos para radiografias intrabucais com localizadores de extremidade de saída aberta para posicionar 0 feixe elimitar a distância foco-pele, sendo 56,3\% dos localizadores tipo cilindro aberto plástico, e $27,2 \%$, cilindro aberto blindado. Dados semeIhantes foram encontrados por Salineiro \& $\mathrm{Ca}$ pelozza ${ }^{24}$ e Spyrides et al. ${ }^{26}$, sendo superiores aos encontradospor Capelozza \& Álvares ${ }^{22}$, nos quais $51 \%$ dos aparel hos possuíam localizadores cilíndricos; eaos de Mutyabule \& Whaites ${ }^{17}$, nos quais somente $39 \%$ possuíam cilindro plástico aberto. Todavia, 10,7\% dos aparel hos têm localizador cônico, não sendo este tipo apropriado por haver interação do feixe primário com a extremidade de saída do localizador (art.5 $§ 7$ alínea e) 7 . Valor semelhante foi encontrado por Tosoni et al. ${ }^{30}$, que relataram que $9,7 \%$ dos profissionais ainda utilizam localizadores cônicos.

Quanto ao tipo de marcador de tempo de exposição, é indicado pelo Ministério da Saúde 0 tipo eletrônico, sendo ideal o uso do marcador digital, pela maior precisão na marcação do tempo, já que um marcador de tempo eficiente é essencial na prevenção de superexposição ${ }^{11}$. Dos dentistas entrevistados, 44,7\% utilizavam o marcador eletrônico e 34\% faziam uso do marcador digital, total izando 78,7\% de profissionais seguindo as normas vigentes. $\mathrm{Na}$ literatura, Capelozza \& Álvares ${ }^{22}$ registraram $86 \%$ de marcadores de tempo eletrônico; Spyrides et al. ${ }^{26}$, $82 \%$; e Jacobs 
et al. ${ }^{18}, 65 \%$. Entretanto, Mutyabule $\&$ Whaites ${ }^{17}$ relataram que $51 \%$ dos marcadores de tempo em Uganda, África, eram do tipo mecânico, número superior aos $18,4 \%$ encontrados nesta pesquisa.

A utilização do sistema de acionamento de disparo com retardo, apesar deser defendido por Pereira ${ }^{31}$, éhoje de uso proibido, segundo 0 art.5 $\S 8$ alínea $\mathrm{h}$ das Diretrizes de Proteção do M inistério da Saúde 7 . Apesar disso, 44,7\% dos profissionais ainda fazem uso deste dispositivo durante 0 exame radiográfico. Esse número é ainda menor que os $92 \%$ encontrado por Spyrides et al. ${ }^{26}$ no Rio de Janeiro.

0 processamento radiográfico éuma das etapas mais importantes para obtenção de exames com qualidade; assim, métodos criteriosos devem ser adotados durante a sua execução. Diferentemente de Goren et al. ${ }^{12}$, que encontraram mais de $50 \%$ dos consultórios com processamento radiográfico automatizado, e de Svenson \& Petersson ${ }^{14}$, com $89 \%$ dos processamentos desta forma, mas semelhante aos 100\% achados por Spyrides et al . ${ }^{26}$ e os $94,4 \%$ relatados por Tosoni et al. ${ }^{30}$, todos os profissionais aqui entrevistados utilizavam o método manual derevelação, sendo $68 \%$ das câmaras escuras portáteis confeccionadas em acrílico vermelho e somente $32 \%$ em material opaco, como estabel ece as normas (art.5 $\S 6$ alínea b $)^{7}$.

Sabe-se ainda que o método tempo/temperatura é o mais indicado para o processamento, sendo proibido qualquer inspeção visual do filme durante os processamentos manuais (art.5 $\S 12$ alínea d) ${ }^{7}$. A pesar disto, $67 \%$ dos cirurgiõesdentistas entrevistados ainda utilizam-se deste artifício, sendo que dos 35\% que não receberam a visita da Vigilância, 83,3\% revelam por este método, demonstrando a importância desta instituição na conscientização dos profissionais. 0 método visual também predominou nos trabaIhos de Capelozza \& Álvares ${ }^{22}$, com 97,2\%; Salineiro \& Capelozza ${ }^{24}$, com $100 \%$; Spyrides et al. ${ }^{26}$, com 83\%; Barbosa \& Gewehr27, com 60\%; e Tosoni et al.$^{30}$, com $81,6 \%$ dos processamentos.

Entretanto, apesar de $33 \%$ dos cirurgiões afirmarem revelar pelo método tempo-temperatura, apenas $28,2 \%$ fazem uso de cronômetro, termômetro e tabela de revelação. Ou seja, 4,8\% dos profissionais optam por revelar por uma técnica da qual não dispõem de todo material necessário para tal. M elhor resultado foi o encontrado por Farman \& Hines ${ }^{9}$, na América do Norte, em que $93 \%$ dos profissionais faziam monitoração diária da temperatura das soluções processadoras.
A utilização de avental plumbífero, com no mínimo 0,25mm dechumbo equegarantaa proteção do tronco dos pacientes, está bastante difundida entre os profissionais das áreas da saúde. Assim como os dados relatados por Farman $\&$ H ines ${ }^{9}$, M onsour et al. ${ }^{10}$, Goren et al. ${ }^{12}$, Salineiro \& Capelozza ${ }^{24}$, Spyrides et al..$^{26}$ e Silveira et al. ${ }^{28}$, a grande maioria $(98,1 \%)$ dos cirurgiõesdentistas avaliados na presente pesquisa afirmaram ser rotina o uso deste aparato, demonstrando um acentuado aumento no seu uso, quando comparado aos 48,6\% encontrados por Garcez Filho et al. ${ }^{23} \mathrm{em}$ 1990, na mesma cidade. No entanto, o uso de protetores dechumbo para tireóide apresenta uma menor adesão por parte dos entrevistados, sendo observado em $70 \%$ dos consultórios. Farman \& Hines ${ }^{9}$ encontraram 97\% dos entrevistados utilizando este protetor; $M$ onsour et al. ${ }^{10}$ obtiveram $50 \%$ de respostas positivas; Goren et al. ${ }^{12}, 60 \%$; e Silveira et al..$^{28}$ encontraram $66,6 \%$. Salineiro \& Capelozza ${ }^{24}$ e Spyrides et al. ${ }^{26}$ não encontraram nenhum protetor de tireóide nos consultórios dos profissionais por eles pesquisados; Barbosa \& Gewehr ${ }^{27}$ encontraram 42\%; Tosoni et al. ${ }^{30}, 18,9 \%$; e llgüy et al. ${ }^{20}$, apenas 3,7\%.

A proteção do profissional também tem sido enfatizada, uma vez que o poder de penetração das radiações ionizantes é inversamente proporcional ao quadrado da distância, sendo essencial a adoção de medidas eficazes que atenuem a sua penetração. Dessa forma, é necessário que, em exames intrabucais em consultórios, o operador mantenha-se a uma distância de, pelo menos, dois metros do tubo e do paciente durante as exposições. Se a carga de trabalho for superior a 30mAmin por semana, o operador deve manterse atrás de uma barreira protetora com uma espessura de, pelo menos, 0,5mm equivalentes ao chumbo (art.5 $\S 9$ alínea b) ${ }^{7}$. Foi encontrado no presente estudo que $7,8 \%$ dos dentistas utilizam o biombo como forma de proteção; $27,2 \%$ relatam se proteger atrás de uma parede de alvenaria, saindo da sala durante o exame, não sendo o mais indicado, pois o operador deve observar e ouvir o paciente durante as exposições (art. 5 §8 alínea g) ${ }^{7} ; 32 \%$ utilizam avental plumbífero, pois em seus consultórios não é possível distanciar-se o suficiente do tubo; e 33\% afirmaram utilizar do distanciamento como forma de autoproteção; todavia, 38\% dos consultórios destes profissionais não permitem um distanciamento de mais de dois metros.

Trabalhos anteriores demonstram concordância com os fatos aqui relatados. M onsour et 
al. ${ }^{10}$ encontraram que $9,1 \%$ dos profissionais utilizavam biombo ou outro tipo de barreira protetora e que considerável número ficava a menos de dois metros de distância da fonte. Salineiro \& Capelozza ${ }^{24}$ apontaram que $90 \%$ saíam da sala durante o exame, e Silveira et al. ${ }^{28}$, que $92,9 \%$ utilizavam este artifício, número maior que os $25 \%$ encontrados por Jacobs et al. ${ }^{18}$.

Uma forma muito eficaz no controle individual do nível de radiação recebida é através da monitoração pessoal, no qual dosímetros são usados para mensurar a dose de radiação a que o indivíduo está exposto em um período predeterminado. N este estudo, apesar de $9,8 \%$ dos cirurgiões-dentistas da cidadenão saberem do que se trata a monitoração pessoal, foi encontrado que $8,7 \%$ fazem uso deste dispositivo, resultado superior aos 3,6\% encontrados por Ilgüy et al. ${ }^{20}$.

Como proteção do público em geral, éindicado pelo M inistério da Saúde ${ }^{7}$ que as salas equipadas com aparelhos de raios $X$ devem dispor de sinalização visível nas portas de acesso, contendo o símbolo internacional da radiação ionizante acompanhado da inscrição "raios $X$, entrada restrita", e de quadro com orientações de proteção radiológica. É importante também que a sala de espera para os clientes esteja protegida de radiações secundárias. Segundo M arques Jr. et al. ${ }^{32}$, as divisórias de madeira apresentaram elevada transmissão da radiação (entre 53 e 89\%); as paredes de tijolos com furos, assim como a de $5 \mathrm{~cm}$ de concreto, revelaram baixa transmissão de radiação, entre 0,00114 e 0,00789\%, val ores que indicam uma proteção relativa da ordem de 10.000 a 100.000 vezes; por fim, as barreiras de tijolos ma- ciços, $10 \mathrm{~cm}$ de concreto e chumbo apresentaram as mais baixas transmissões observadas, variando entre $0,00084 \mathrm{e} 0,00051 \%$, o que equivaleauma proteção de 100.000 a 1.000.000 de vezes.

Em Aracaju, apenas 1,9\% dos consultórios avaliados apresentam algum tipo de sinalização de radiação. Com relação à parede que separa a sala de espera do consultório, ou sala onde está instalado o equipamento, esta é de madeira em $33 \%$ dos casos, de alvenaria em $64 \%$ e em 1,9\% dos consultórios não havia qualquer divisória. Garcez Filho et al..$^{23}$ haviam encontrado $71,4 \%$ de divisórias de madeira, demonstrando uma tendência de adequação dos consultórios às normas de proteção, como o encontrado por Salineiro \& Capelozza ${ }^{24}$, com 93\% de divisórias de alvenaria.

\section{Conclusão}

A partir da análise dos resultados encontrados, pôde-se concluir que uma minoria dos profissionais tem conhecimento da existência das normas vigentes; em relação à utilização dos apareIhos de raios $X$, os profissionais ainda não se enquadraram corretamente às exigências feitas pelos órgãos competentes; os profissionais têm mostrado alta aceitabilidade e reprodutibilidade das diretrizes de proteção em relação ao paciente; no entanto, atendem somente em parte aos métodos de proteção pessoal; há necessidade de campanhas edu cativas, a fim de dissi par os questionamentos dos profissionais, desmistificando as normas de proteção, além de evitar sanções por parte dos órgãos fiscalizadores. 


\section{Colaboradores}

M FB M elo trabalhou na concepção teórica, análisee redação final do texto. SLSM elo realizou as entrevistas e a transcrição das respostas, além de participar da discussão dos achados e da redação do texto.

\section{Agradecimentos}

Pesquisa desenvolvida com o apoio do Programalnstitucional deBolsas de Iniciação Científica do Conselho Nacional de Desenvolvimento $\mathrm{Ci}$ entífico e Tecnológico (PIBIC-CN Pq), em parceria com a Coordenação de Pesquisa da Universidade Federal de Sergipe (COPES-UFS), através da concessão de bolsa de iniciação científica.

\section{Referências}

1. Gomes RO, Gomes RD. A radiologia na odontologia - efeitos biológicos e medidas de proteção. Rev Bras Odontol 1983; 6:7-11.

2. Campos AA, Watanabe PCA, Pardini LC, Tamburus JR. Avaliação do diâmetro do feixe útil de radiação de aparelhos de raios $X$ odontológicos. Rev Odontol Univ São Paulo 1995; 9(4):293-297.

3. Abbott P. Are dental radiographs safe? Aust D ent J 2000; 45(3):208-213.

4. Watanabe PCA, Pardini LC, Arita ES. Discussão das diretrizes de proteção radiológica em radiodiagnóstico médico e odontológico. Rev Assoc Paul Cir Dent 2000; 54(1):64-72.

5. Smith NJD. Risk assessment: the philosophy underlying radiation protection. Int Dent J 1987; 37(1):43-51.

6. Smith NJD. Radiation protection. The administrative and legal framework in the United Kingdom. $\mathrm{Br}$ Dent J 1978; 144(2):47-49.

7. Brasil. Portaria no 453 de 01 de junho de 1998. Diretrizes de proteção radiológica em radiodiagnóstico médico e odontológico. Diário Oficial da União 1998; 2 jun.

8. Farman AG, Nortjé CJ. Practical radiology education in South African Dental Schools, 1981. J Dent Assoc S Afr 1981; 36(10):685-688.

9. Farman AG, Hines VG. Radiation safety and quality assurance in North American dental schools. J Dent Educ 1986; 50(6):304-308.

10. M onsour PA, Kruger BJ, Barnes A, Sainsbury A. $M$ easures taken to reduce $X$-ray exposure of the patient, operator, and staff. Aust Dent J 1988; 33(3):181-192.

11. Mageean JF. Dental radiology in Northern Ireland: radiation protection. Community $\mathrm{D}$ ent $\mathrm{H}$ ealth 1989; 6(4):415-419.

12. Goren AD, Sciubba JJ, Friedman R, Malamud $H$. Survey of radiologic practices among dental practitioners. Oral Surg Oral Med Oral Pathol 1989; 67(4):464-468.

13. Geist JR, Katz JO. The use of radiation dose-reduction techniques in the practices of dental faculty members. J Dent Educ 2002; 66(6):697-702.

14. Svenson B, Petersson A. Questionnaire survey on the use of dental $X$-ray film and equipment among general practitioners in the Swedish Public Dental Health Service. Acta Odontol Scand 1995; 53(4):230-235.

15. Svenson B, Söderfeldt B, Gröndahl HG. Attitudes of Swedish dentists to the choice of dental X-ray film and collimator for oral radiology. Dentomaxillofac Radiol 1996; 25(3):157-161.

16. Svenson B, Gröndahl HG, Söderfeldt B. A logistic regression model for analyzing the relation between dentists' attitudes, behavior, and knowledge in oral radiology. Acta Odontol Scand 1998; 56(4):215-219.

17. M utyabule TK, Whaites EJ. Survey of radiography and radiation protection in general dental practice in U ganda. Dentomaxillofac Radiol 2002; 31(3):164169.

18. Jacobs R, Vanderstappen M, Bogaerts R, Gijbels F. Attitude of the Belgian dentist population towards radiation protection. Dentomaxillofac Radiol 2004; 33(5):334-339. 
19. Stavrianou K, Pappous G, Pallikarakis N. A quality assurance program in dental radiographic units in western Greece. Oral Surg Oral Med Oral Pathol Oral Radiol Endod 2005; 99(5):622-627.

20. Ilgüy D, Ilgüy M, Dinçer S, Bayirh G. Survey of dental radiological practice in Turkey. Dentomaxillofac Radiol 2005; 34(4):222-227.

21. M elo M FB, Freitas A, Abramowicz M. Condições de utilização dos aparelhos de raios $X$ e medidas de prevenção das radiações $X$, por cirurgiões-dentistas na cidade de São Paulo. Rev Fac Odontol U niv São Paulo 1985; 23(2):89-105.

22. Capelozza ALA, Álvares LC. Avaliação das condições de radioproteção em consultórios odontológicos na cidade de Bauru - SP. Rev Assoc Paul Cir Dent 1989; 43(4):193-195.

23. Garcez Filho JA, Rocha APB, Oliveira MLB. M eios de proteção dos raios $X$ : análise comparativa das condições de utilização e proteção radiológica entre profissionais da Odontologia. Rev Gauc Odontol 1990; 38(3):177-180.

24. Salineiro SL, Capelozza ALA. Avaliação das condições de radioproteção em cem consultórios odontológicos da cidade de Araçatuba, Estado de São Paulo. Rev Fac Odontol Bauru 1997; 5(1/2):65-70.

25. Bóscolo FN, Braga Jr DL, Gonçalves A, Filho AM. Contribuição ao estudo da quilovoltagem nominal preconizada e do tempo de exposição de diferentes modelos de aparelhos de raios $X$ odontológicos. Rev Odontol Univ São Paulo 1997; 11:43-49.

26. Spyrides KS, Oliveira AEF, Almeida SM, Bóscolo FN. Avaliação do controle de qualidade e proteção radiológica na cidade do Rio de Janeiro. Rev Bras Odontol 2001; 58(5):321-323.

27. Barbosa OD, Gewehr PM. Programa para avaliação e controle da utilização de raios $X$ em clínicas odontológicas. In: Memórias II Congresso Latinoamericano de Ingeniería Biomédica; 2001; Cuba.

28. Silveira M M F, M onteiro IS, Brito SA. Avaliação da utilização dos meios de radioproteção em consultórios odontológicos em Olinda/PE. Odontol Clincient 2005; 4(1):43-48.

29. Bridgman JB, Campbell DJ. An upgrade on dental radiology: quality and safety. N Z Dent J 1995; 91(403):16-21.

30. Tosoni GM, Campos DM, Silva MR. Freqüência de cirurgiões-dentistas que realizam exame radiográfico intrabucal e avaliação das condições para a qualidade do exame. Rev Odontol U nesp 2003; 32(1):25-29.

31. Pereira GG. Considerações sobre a utilização da radiação ionizante em odontologia. Odontol Mod 1990; 17(9):10-13.

32. M arques Jr A, Fontoura HES, Souto SLL. Avaliação das barreiras à radiação X. RGO 1991; 39(4):273-275.

Artigo apresentado em 31/10/2006

Aprovado em 04/06/2007

Versão final apresentada em 11/07/2007 